\title{
A NEW JUNCTION PARAMETERS DETERMINATION USING THE DOUBLE EXPONENTIAL MODEL
}

\author{
S. DIB ${ }^{\mathrm{a}}$, A. KHOURY ${ }^{\mathrm{a}}$, F. PÉLANCHON ${ }^{\mathrm{b}}$ and P. MIALHE ${ }^{\mathrm{b}, *}$ \\ ${ }^{a}$ Centre d'Etudes Fondamentales, Université de Perpignan, 52 Avenue de Villeneuve, 66860 \\ Perpignan Cedex, France; ${ }^{\mathrm{b}}$ LP2A, Département de Physique, Université Libanaise-Faculté de \\ Sciences II, BP 90656 Jdeidet, Liban
}

(Received 15 November 2001; In final form 3 December 2001)

A double exponential model is used to characterize the junction properties of microelectronic devices. A method is developed to extract the physical junction parameters from the current-voltage characteristics. The influence of the start values used for the computing processes are considered and the accuracy is discussed from results of simulated extractions. An experimental test considers the emitter-base junction of bipolar transistors.

Keywords: Characteristic; Junction; Parameters; Extraction; Modeling

\section{INTRODUCTION}

The p-n junction current-voltage ( $I-V)$ characteristics have been extensively studied and many mathematical models, based on the physics of semiconductors, have been developed. The $I-V$ characteristic of a junction can be described [1-8] by an implicit equation:

$$
I=I_{\mathrm{o} 1}\left(\exp \left(\frac{V-R_{S} I}{V_{T}}\right)-1\right)+I_{\mathrm{o} 2}\left(\exp \left(\frac{V-R_{S} I}{A V_{T}}\right)-1\right)+\frac{V-R_{S} I}{R_{\mathrm{sh}}}
$$

known as the double exponential model. $V_{T}=\mathrm{k} T / q$ is the thermal voltage. $A$ is the junction quality factor: it is commonly considered to be related to the recombination processes, mainly in the space-charge region of the junction $[1,3,6]$. A usually varies between 1 and $2 . R_{S}$ and $R_{\mathrm{sh}}$ are the series and the shunt resistances in the equivalent circuit. $I_{\mathrm{o} 1}$ and $I_{\mathrm{o} 2}$ are the components of the diode reverse saturation current (respectively the diffusion current in the bulk of the junction $I_{01}$ and the recombination $I_{02}$ current $[1,3,6]$ ).

The five parameters $A, R_{s}, R_{\mathrm{sh}}, I_{\mathrm{o} 1}, I_{\mathrm{o} 2}$ are related to internal properties of the electronic device and have been used in modeling studies. They are of interest for the description of carrier transport phenomena and power losses in operating junctions, and for quantifying the quality of diodes and transistors structures.

\footnotetext{
* Corresponding author. E-mail: mialhe@univ-perp.fr
} 
This work presents a new method to extract the junction parameters $A, R_{s}, R_{\mathrm{sh}}, I_{\mathrm{o} 1}, I_{\mathrm{O} 2}$ related to the double exponential model, from the experimental $(I-V)$ characteristic. New parameters are introduced for computing processes and a least square fitting of the $I-V$ characteristic is performed, using the high voltage region of the curve. The accuracy of the method is discussed at length, both with "theoretical" characteristics and experimental emitter-base junction characteristics of transistors. It is pointed out that the method is accurate and easy to implant in any technological process for quality control.

\section{THEORETICAL METHOD}

The method consists in firstly determining $R_{\mathrm{sh}}$ and $R_{S}$, then in replacing the unknown parameters, $A, I_{01}$ and $I_{02}$ by some quantities allowing to use the least square method.

The determination of the series and shunt resistances is performed from the junction $I-V$ characteristics obtained under the form of $N$ experimental couples $(I(k), V(k))_{1 \leq k \leq N}$ by using any acquisition system.

Since, near the origin, each $\exp \left(\left(V-R_{S} I\right) / V_{T}\right)$ term is very close to $1, R_{\mathrm{sh}}$ may be determined, for low $V$ and $I$ values, by the approximation :

$$
\frac{\mathrm{d} I}{\mathrm{~d} V}=\frac{1}{R_{\mathrm{sh}}}
$$

A first value $\rho_{s}$ of $R_{s}$ can be easily determined by the single diode model [4] and used in our process.

From Eq. (1), the theoretical approach considers $I$ expressed as $I=I_{1}+I_{2}+I_{3}$ and introduces the quantities: $I_{1}=I_{\mathrm{o} 1} \exp \left(\left(V-R_{S} I\right) / V_{T}\right), I_{2}=I_{\mathrm{o} 2} \exp \left(\left(V-R_{S} I\right) / A V_{T}\right)$ and $I_{3}=\left(V-R_{S} I\right) / R_{\mathrm{sh}}$.

These expressions obviously yield:

$$
\frac{V-R_{S} I}{V_{T}}=\ln \left(\frac{I_{1}}{I_{\mathrm{o} 1}}\right)=\ln \left[\left(\frac{I_{2}}{I_{\mathrm{o} 2}}\right)^{A}\right]
$$

then:

$$
\frac{I_{1}}{I_{2}^{A}}=\frac{I_{\mathrm{o} 1}}{I_{\mathrm{o} 2}^{A}} .
$$

Let us then consider the (unknown) parameter $D$ defined by the following relation:

$$
D=\frac{I_{1}}{I_{2}^{A}}=\frac{I_{\mathrm{o} 1}}{I_{\mathrm{o} 2}^{A}} .
$$

Using Eq. (5) and the equality : $I-I_{3}=I_{1}+I_{2}, I_{2}$ appears as a solution of the easily solvable (by the Newton's method) equation:

$$
I-I_{3}=I_{2}+D \cdot I_{2}^{A}
$$


Since $V_{T} \cdot \ln I_{2}^{A}=V_{T} \cdot \ln I_{\mathrm{o} 2}^{A}+V-R_{S} I$, the function $V^{\prime}=V-V_{T} \cdot \ln \left(I_{2}^{A}\right)$ appears as a linear function of $I$ :

$$
\begin{gathered}
\qquad \begin{aligned}
V^{\prime} & =a I+b \\
\text { where }: a & =R_{s} \text { and } b=-V_{T} \cdot \ln \left(I_{\mathrm{o} 2}^{A}\right) .
\end{aligned}
\end{gathered}
$$

This function $V^{\prime}(I)$ is linear when both $A$ and the deduced parameter $D$ are exactly related to Eq. (1). Section 3 details the use of the least square method, applied to the $N$ experimental couples $(I(k), V(k))$ and to the corresponding $I_{3}, I_{2}$ and $I_{1}$ values in order to find out $A$ and $D$.

Once the actual values of $A$ and $D$ have been determined through the least square method (that determines both $a$ and $b$, Eq. (8)), Eqs. (5) and (8) give:

$$
I_{\mathrm{o} 2}=\exp \left(\frac{-b}{V_{T} A}\right) \text { and } I_{\mathrm{o} 1}=D I_{\mathrm{o} 2}^{A} \text {. }
$$

\section{LEAST SQUARE METHOD APPLIED TO THE EXPERIMENTAL $I-V$ CHARACTERISTICS}

Our computing process consists in adjusting the values of $A$ and $D$ so that the experimental representation of Eq. (7) is as linear as possible. For that purpose, a reference table of 15625 values of $(A, D)$ is built as follows. $A$ is arithmetically varied from 1 to 2.25 by step of 0.01 , and $D$ is geometrically varied from $10^{-3}$ to $10^{12}$ by step of 1.318 (see Appendix 1 for the boundary values of $A$ and $D$ ).

The program picks up any value of $(A, D)$ in this reference table. For a given $(A, D)$ value, the least square method is applied as follows. The experimental $I-V$ characteristics is associated to the initial table $(I(k), V(k)), 1 \leq k \leq N$. The two resistance values can be calculated from section 2.3. Then, the $N$ corresponding values of both $I-I_{3}$ and $I_{2}$ are calculated. Finally, the $N$ values of $V^{\prime}=V-V_{T} \cdot \ln \left(I_{2}^{A}\right)$ are computed.

Starting from an original (experimental) $I-V$ characteristic, our method successively extracts the (estimated) values of $R_{s}, R_{\mathrm{sh}}, A, D, I_{\mathrm{o} 1}$ and $I_{02}$. Then, Eq. (1) allows to build an estimated $I-V$ characteristic.

The well-known function $Q[5,10]$, defined as $Q=\left[1 / N \sum_{k=1}^{k=N} d(k)^{2}\right]^{1 / 2}$, where $d(k)$ is the distance between the points of the calculated characteristic and the experimental points, in relative units [5], has been used to compare the two characteristics. A calculated value of $Q$ lower than $5 \times 10^{-3}$ corresponds to a good fitting. In order to provide a faster estimation of $A$ and $D, Q$ is replaced by the function $L Q=\ln (1 / Q)$. The choice of the best $Q$ value (close to 0 ) determines the sought values of $A$ and $D$, called $A m$ and $D m$.

\section{CRITICAL STUDY OF THE METHOD}

The accuracy of the different steps of our method depends on the accuracy of the calculus of $R_{s}, R_{\mathrm{sh}}$ and $I_{2}$ (section 2).

\subsection{Influence of $R_{S}$}

The series resistance $R_{S}$ is calculated through a two step process. A first determination $\rho_{S}$ of the series resistance $R_{s}$ is obtained from the single diode model [4]. This value $\rho_{s}$ is then used in the least square method providing a statistically estimated value of the parameter " $a$ " that is then assimilated to $R_{s}$. 


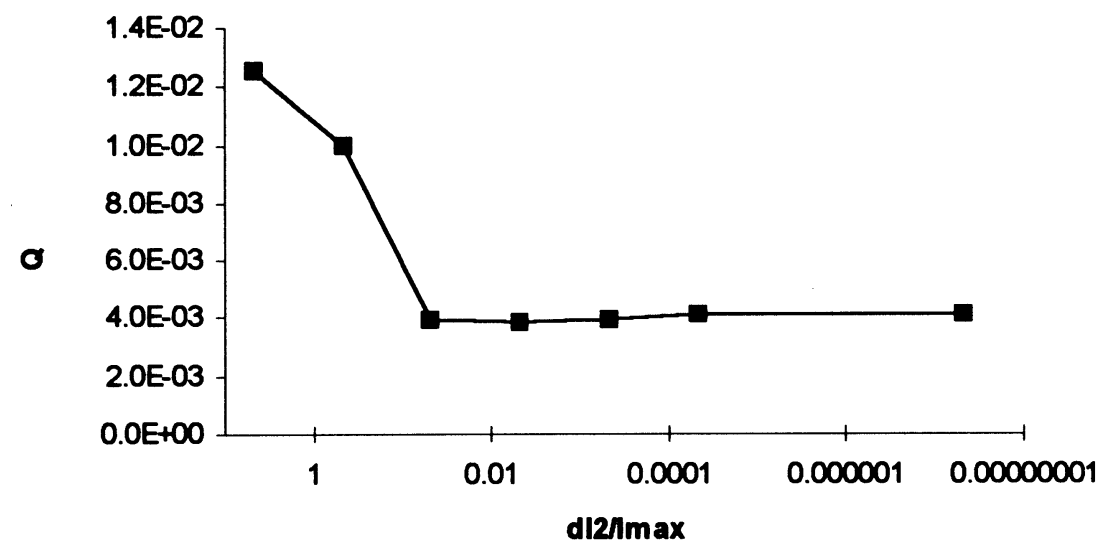

FIGURE $1 Q$ function versus the ratio $\mathrm{d} I_{2} / I_{\max }$ for the emitter-base junction characteristic of a $2 \mathrm{~N} 2904 \mathrm{~A}$ transistor.

In order to test the influence of this choice of $\rho_{s}$ on the accuracy of the fit between the two characteristics, this method has been performed with a theoretical $I-V$ characteristic for various $R_{\mathrm{sh}}$ values. The ratio $\rho_{s} / R_{S}$ is mostly lower than 1.4 and very close to 1 as soon as $R_{\text {sh }}>300 \mathrm{ohm}$. $Q$ stays lower than $5 \times 10^{-3}$ for a large range of the shunt resistance value. Besides, the fit (related to low $Q$ values) is all the better as the shunt resistance value is high. Furthermore, when the shunt resistance is beyond 50,000 ohm, the influence of the ratio $\rho_{s} / R_{s}$ on the $Q$ variations is negligible: thus, in this case, the choice of $\rho_{s}$ as initial value in our process leads to a good accuracy.

\subsection{Influence of $I_{2}$}

In our computed process, $I_{2}$ was calculated, by Newton's method, through Eq. (6), with a precision lower than $10^{-9}$. But, since $V^{\prime}$ depends on $I_{2}\left(\mathrm{~d} V^{\prime}=\left(-A V_{T} / I_{2}\right) \mathrm{d} I_{2}\right)$ and because of possible very low values of $I_{2}$, potential discrepancies could appear in the computation of $V^{\prime}$.

In the method, the choice of $\mathrm{d} I_{2}$ depends on the maximum value $I_{\max }$ of the current $I$ on the (theoretical or experimental) characteristic : $\mathrm{d} I_{2}=I_{\max } \cdot 10^{-5}$. In our different trials, $Q$ stayed lower than $5 \times 10^{-3}$ for $\mathrm{d}_{2}<0.05 \cdot I_{\max }$ (Fig. 1).

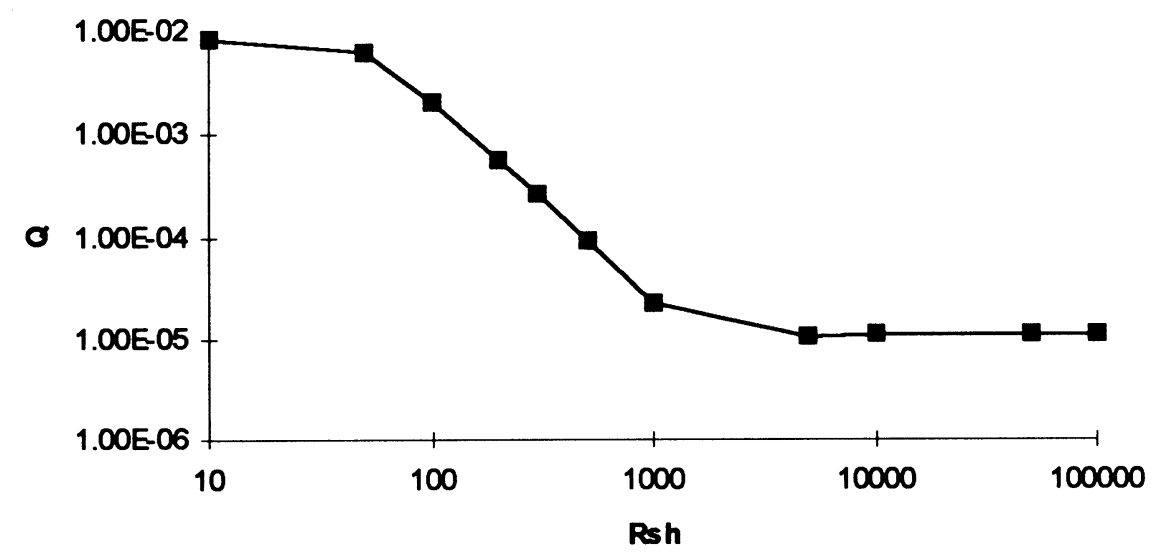

FIGURE $2 Q$ function versus the shunt resistance value $R_{\text {sh }}$ for a theoretical characteristic $\left(R_{s}=5 \Omega\right.$; $\left.A=1.7 ; I_{\mathrm{o} 1}=10^{-12} \mathrm{~A} ; I_{\mathrm{o} 2}=10^{-9} \mathrm{~A}\right)$. 


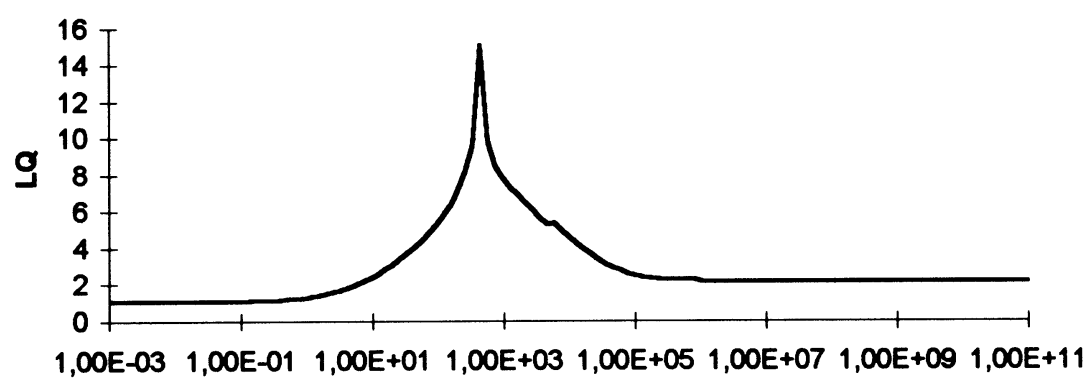

D

FIGURE 3 Zoom on $L Q$ variations versus $D$, while $D$ varies around $D_{m}$ for a theoretical characteristic $\left(R_{\mathrm{sh}}=60,000 \Omega ; R_{S}=1.5 \Omega ; A=1.65 ; I_{\mathrm{o} 1}=2 \times 10^{-13} \mathrm{~A} ; I_{\mathrm{o} 2}=5 \times 10^{-10} \mathrm{~A}\right)$.

\subsection{Influence of $\boldsymbol{R}_{\mathrm{Sh}}$}

The determination of $R_{\mathrm{sh}}$ implies the $I-V$ characteristic in the vicinity of the origin $(V<0.5 \mathrm{~V})$. The accuracy of the $I-V$ measurement influences the accuracy $\mathrm{d} R_{\mathrm{sh}}$ on $R_{\mathrm{sh}}$. When $R_{\text {sh }}>200 \mathrm{ohm}$ (usual values for good quality junctions), $Q<5 \times 10^{-3}$ (Fig. 2).

\section{THEORETICAL TEST}

As an example, the method has been applied to a theoretical $I-V$ characteristic obtained from Eq. (1) with given arbitrary values of the parameters $R_{s}, R_{\mathrm{sh}}, A, I_{\mathrm{o} 1}$ and $I_{\mathrm{o} 2} \cdot\left(R_{\mathrm{sh}}=60,000 \Omega\right.$; $\left.R_{s}=1.5 \Omega ; A=1.65 ; I_{\mathrm{o} 1}=2 \times 10^{-13} \mathrm{~A} ; I_{\mathrm{o} 2}=5 \times 10^{-10} \mathrm{~A}\right)$.

The program yields $A m=1.65$ and $D m=440$. Figure 3 displays $L Q$ zoom around $A m$ and $D m$ values. Value of $L Q$ about 12 correspond to $Q<7 \times 10^{-6}$ !

Several similar trials have been performed with different initial values of the parameters $R_{s}, R_{\mathrm{sh}}, A, I_{\mathrm{0} 1}$ and $I_{\mathrm{o} 2}$ and led to the same quasi perfect fit between the two characteristics.

Table I shows the results obtained with the same initial parameter values, with three different $R_{\mathrm{sh}}$ values. This table clearly points out the influence of $R_{\mathrm{sh}}$. Furthermore, the accuracy of the method strongly depends on the fact that $R_{\mathrm{sh}}$ is great enough. Practically, most of the junctions are characterized by a shunt resistance $R_{\mathrm{sh}}$ greater than $300 \mathrm{ohms}$ which yields a good accuracy of the results.

TABLE I Estimated Parameter Values Extracted with our Method from a Theoretical Characteristic and for Three Shunt Resistance $R_{\text {sh }}$ Values.

\begin{tabular}{llllrrc}
\hline & & $A$ & $R_{s}(\Omega)$ & \multicolumn{1}{c}{$I_{\mathrm{o} 1}(A)$} & \multicolumn{1}{c}{$I_{\mathrm{o} 2}(A)$} & $R_{\mathrm{sh}}(\Omega)$ \\
\hline Characteristic 1 & Inital values & 1.6 & 0.1 & $5 \times 10^{-15}$ & $5 \times 10^{-11}$ & $10^{5}$ \\
$\left(R_{\mathrm{sh}}\right)=10^{5} \Omega$ & Extracted values & 1.61 & 0.0998 & $5.07 \times 10^{-15}$ & $7.88 \times 10^{-11}$ & 99982 \\
Characteristic 2 & Initial values & 1.6 & 0.1 & $5 \times 10^{-15}$ & $5 \times 10^{-11}$ & $10^{5}$ \\
$\left(R_{\mathrm{sh}}\right)=1000 \Omega$ & Extracted values & 1.62 & 0.104 & $5.06 \times 10^{-15}$ & $5.00 \times 10^{-11}$ & 1000.1 \\
Characteristic 3 & Initial values & 1.6 & 0.1 & $5 \times 10^{-15}$ & $5 \times 10^{-11}$ & $10^{5}$ \\
$\left(R_{\mathrm{sh}}\right)=200 \Omega$ & Extracted values & 1.74 & 0.105 & $5.23 \times 10^{-15}$ & $1.71 \times 10^{-10}$ & 200.10 \\
\hline
\end{tabular}




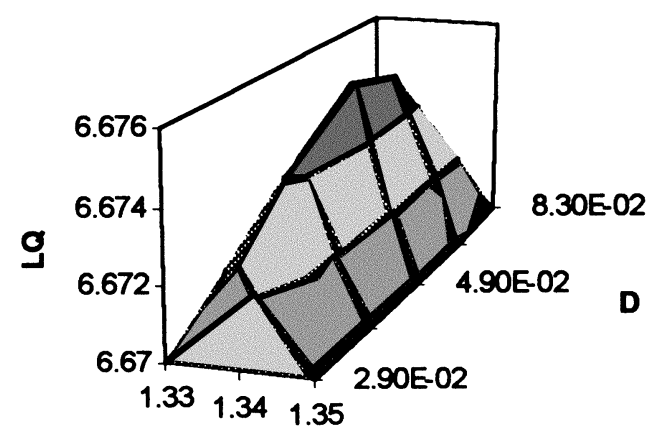

\begin{tabular}{|l|}
\hline $6.674-6.676$ \\
$\square 6.672-6.674$ \\
$\square 6.67-6.672$ \\
\hline
\end{tabular}

FIGURE $4 L Q$ variations versus $A$ and $D$ for an experimental characteristic (base-emitter junction of a 2N2904A transistor).

TABLE II Mean and Standard Deviation of the Results Obtained by Successively using 10 Times the $Q$ Criterion with an Experimental Characteristic (Base-Emitter Junction of a 2N2904A Transistor).

\begin{tabular}{rcccccrr}
\hline & & $A$ & $I_{\mathrm{o} 1}(A)$ & $I_{\mathrm{o} 2}(A)$ & $R_{s}(\Omega)$ & \multicolumn{1}{c}{$R_{\mathrm{sh}}(\Omega)$} & $Q$ \\
\hline $\begin{array}{c}\text { Minimal } Q \\
\text { criterion }\end{array}$ & Mean & 1.30 & $8.10 \times 10^{-17}$ & $3.4 \times 10^{-12}$ & 0.516 & 24838 & $1.12 \times 10^{-3}$ \\
& $\begin{array}{c}\text { Standard } \\
\text { deviation }\end{array}$ & 0.007 & $4.31 \times 10^{-17}$ & $0.63 \times 10^{-12}$ & 0.050 & 4692 & $0.05 \times 10^{-3}$ \\
\hline
\end{tabular}

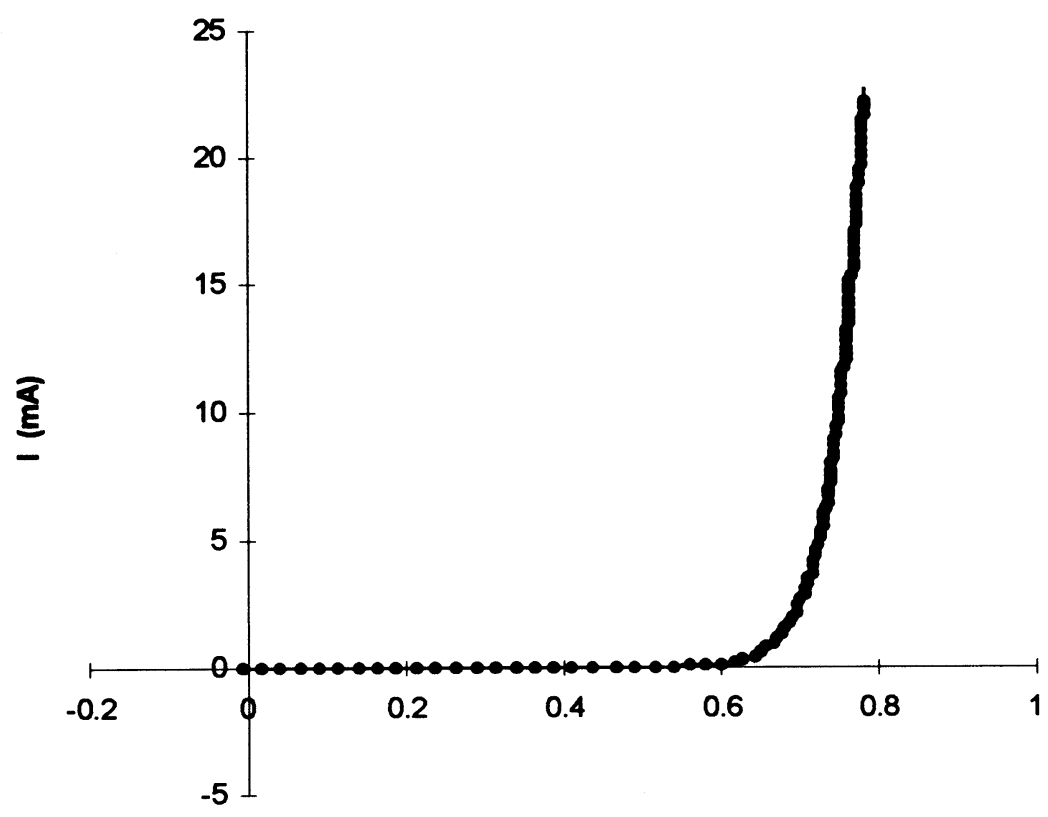

V (V)

- Experimental characteristic Calculated characteristic

FIGURE 5 Experimental characteristic of the emitter-base junction of a 2N2904A transistor (dots) and its fit by our method (line). 


\section{EXPERIMENTAL TEST}

The method has been applied to a physical junction (the base-emitter junction of a transistor $2 \mathrm{~N} 2904 \mathrm{~A})$. The maximum value of $Q(A, D)$ was obtained for $(A=1,34 ; D=0.049)$ (Fig. 4).

Table II points out a very low standard deviation value. Figure 5 represents the experimental $I-V$ characteristic and the calculated characteristic obtained with the estimated values of the parameters $R_{s}, R_{\mathrm{sh}}, A, I_{\mathrm{o} 1}$ and $I_{\mathrm{o} 2}$ through Eq. (1): the two curves are quasi similar.

\section{CONCLUSION}

This paper presents a new method to extract the p-n junction parameters from a description of the current-voltage characteristic with a two exponential model. The influence of the start values used for the computing processes are considered and the accuracy is discussed from results of simulated extractions. An experimental test considers the emitter-base junction of bipolar transistors. The accuracy, the rapidity of the procedure and the simplicity of implanting the calculation process in any program, make it useful for characterization of microelectronic devices and to quantify the quality of any junction.

\section{References}

[1] Sze, S. M. (1981). Physics of Semiconductors Devices, 2nd edn. Wiley-Interscience.

[2] Sah, C.-T., Noyce, R. N., Shockley, W. (1957). Proc. IRE. 45/9, 1228-1243.67.

[3] Pélanchon, F., Mialhe, P. (1990). Solid-St Electron., 33, 47.

[4] Dib, S., de la Bardonnie, M., Khoury, A., Pélanchon, F. and Mialhe, P. (1999). Active and Passive Elect. Comp, 22, 157.

[5] Charles, J. P., Mekkaoui-Alaoui, I., Bordure, G. and Mialhe, P. (1985). Solid-St Electron., $28,807$.

[6] Pallares, J., Marsal, L. F., Correig, X., Calderer, J. and Alcubila, R. (1997). Solid-St Electron., 41, 17.

[7] Veissid, N., Bonnet, H., Richter, H. (1995). Solid-St Electron., 38, 1937.

[8] Gottschalg, R., Rommel, M., Infield, D. G., Kearney, M. J. (1999). Meas. Sci. Technol., 10, 796.

[9] del Cueto, J. A. (1999). Solar Energy Materials \& Solar Cells, 59, 393.

[10] "Probabilité et Statistique", Cours et Problèmes, Série Schaum, 1991; p. 271 and 274.

\section{APPENDIX 1: DETERMINATION OF THE EXTREME VALUES OF $D$ AND $A$.}

A first approach considers $R_{\text {sh }}$ quasi-infinite (greater than $10^{3} \mathrm{ohm}$ ) : in this case, $I_{3}$ is negligible compared to $I$ along the $I-V$ characteristic.

The parameter $D$ Eq. (5) can theoretically reach any real value. Due to the physical constraints, $D$ can be limited between two values $D_{\min }$ and $D_{\max }$ (see below).

When the value of the ratio $\gamma=I_{1} / I_{2}$ is high, $I_{2}$ can be neglected compared to $I_{1}$ and Eq. (1) corresponds to the one-exponential model with $A$ equal to 1 (ideal junction). On the other hand, when $\gamma$ value is low, $I_{1}$ can be neglected compared to $I_{2}$ and Eq. (1) corresponds to the one exponential model with $A$ much greater than 1 (significant recombination processes).

Then, since our study considers a description with the double exponential model, we are concerned with values of parameter $\gamma$ varying in the range $\left(10^{-2}-10^{2}\right)$ : these values yield respective boundaries for $D$ and $A$. 
From Eq. (5) and the equality $I=I_{1}+I_{2}=I_{2}(\gamma+1)$, we obtain:

$$
D=\frac{\gamma(\gamma+1)^{A-1}}{I^{A-1}}
$$

$$
\text { When } \gamma=10^{2}, D=\frac{\gamma^{A}}{I^{A-1}}=\frac{10^{2 A}}{I^{A-1}}
$$

This latter expression is maximal when both $A=A_{\max }=2$ and $I=I_{\min }$. In this study, $I_{\min }$ is chosen (Appendix 2) to $10^{-7} \mathrm{~A}$. Then, $D_{\max }=10^{11}$. In our calculations, the value $10^{12}$ for $D_{\max }$ was used.

$$
\text { When } \gamma=10^{-2}, D=\frac{10^{-2}}{I^{A-1}}
$$

This latter expression is minimal when $A=A_{\min }=1$ and when $I=I_{\max }$. In this study, $I_{\max }$ is chosen to $1 \mathrm{~A}$. Then, $D_{\min }=10^{-3}$. 

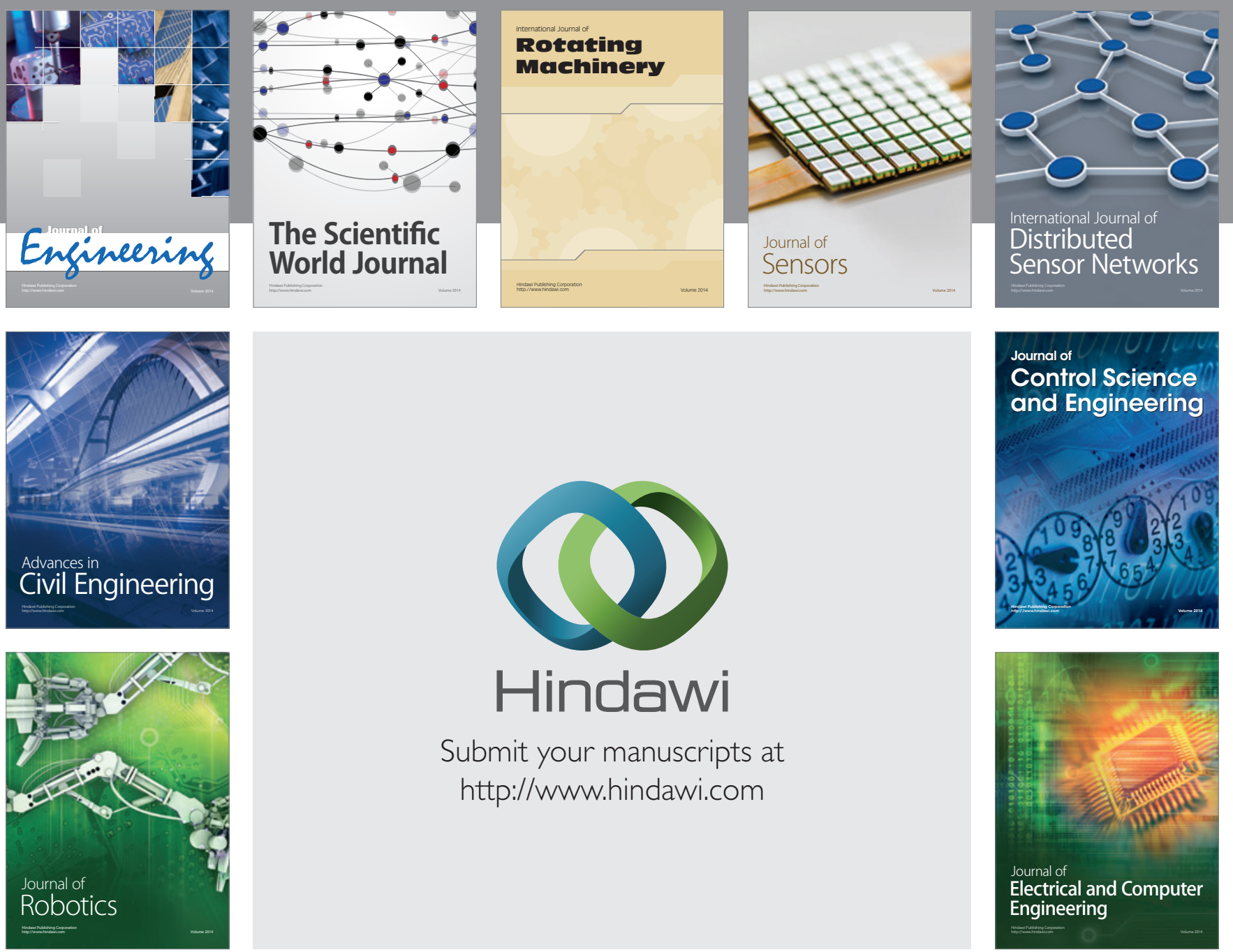

Submit your manuscripts at

http://www.hindawi.com
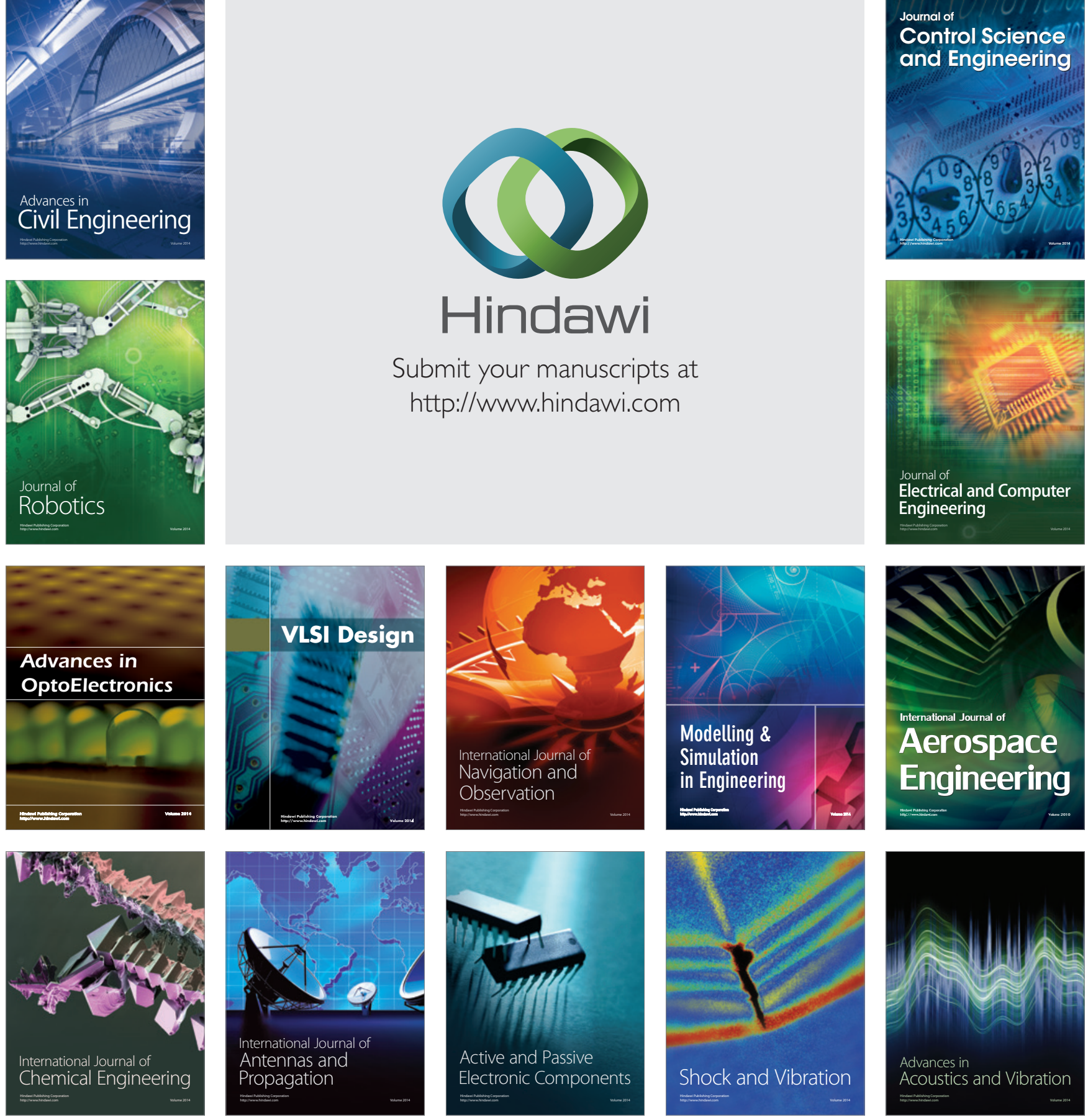